\title{
Cooperative toughening mechanism of nanocrystalline materials by grain rotation and shear-coupled migration of grain boundaries
}

\author{
Jianjun $\mathrm{Li}^{\mathrm{a}}{ }^{\text {,** }}$, Shaohua Chen ${ }^{\mathrm{b}}$ \\ a Department of Engineering Mechanics, School of Mechanics, Civil Engineering and Architecture, Northwestern Polytechnical University, Xi'an, Shanxi, China \\ ${ }^{\mathrm{b}}$ LNM, Institute of Mechanics, Chinese Academy of Sciences, Beijing 100190, China
}

\section{A R T I C L E I N F O}

\section{Article history:}

Received 23 December 2013

Accepted 27 January 2014

Available online 4 February 2014

Keywords:

Nanocrystalline materials

Simulation and modeling

Toughening mechanism

Grain rotation

Shear-coupled migration of grain

boundaries

Stress intensity factor

\begin{abstract}
A B S T R A C T
A theoretical model is proposed to illustrate the cooperative effect of nano-grain rotation and shearcoupled migration of grain boundaries on toughening of nanocrystalline materials. The toughening mechanism is embodied by an effective stress intensity factor near the tip of a mode I crack. The result shows a possibly effective toughening mechanism in nanocrystalline materials.
\end{abstract}

(c) 2014 Elsevier B.V. All rights reserved.

\section{Introduction}

Nanocrystalline (NC) materials usually possess superior strength and hardness but low ductility and toughness as compared with their coarse-grained counterparts due to the inhibition of the conventional dislocation slip in nano-grains [1]. Recently, some NC samples with extraordinary ductility were successfully fabricated [2,3]. Their outstanding mechanical behaviors were attributed to stress-driven nano-grain growth.

Extensive experimental and theoretical studies as well as many molecular dynamics (MD) simulations have shown that shearcoupled migration (SCM) of grain boundaries (GBs) and nanograin rotation (NGR) are two main modes of nano-grain growth behavior in both bicrystals and nano-polycrystallines [4-11]. For example, the MD and phase field crystal simulations studies of Trautt et al. have clearly shown that SCM exists for both symmetrical and asymmetrical GBs [7]. The above coupled motion was further observed in their experiments for Al bicrystals by Gorkaya et al. $[8,9]$. Moreover, there exists a critical misorientation parameter that represents the crossover from SCM to NGR [12]. Recent studies in experiments and MD simulations as well as mesoscale simulations showed that the two modes were intricately interplayed [4,13-16]. For example, Gorkaya et al. have successfully

\footnotetext{
* Corresponding author.

E-mail addresses: jianjunli.mech@gmail.com, mejjli@nwpu.edu.cn (J. Li).
}

discovered a coupling behavior between SCM and rotation in high purity Al bicrystals due to the twist component of the boundary in their experiments [15]. In the MD simulations of an isolated cylindrical grain in copper, Trautt and Mishin disclosed a dislocation propagation mechanism by a chain of dislocation reactions along the curved GB in order to account for the cooperation of the migration process and grain rotation [16]. In addition, a molecular static simulation conducted by Sansoz and Dupont [17] for a $7 \mathrm{~nm}$ grain size nanocrystalline Al thin film under indentation at absolute zero $(0 \mathrm{~K})$ has demonstrated the following coupling behavior of the two modes, i.e., under an externally applied load, NGR occurs first, which leads to a decrease of misorientation parameter $\theta_{0}$, subsequently, SCM appears. This coupling behavior has also been predicted by the theoretical model proposed by Li et al. [12]. However, the detailed coupling mechanisms are complicated due to the multiplicity of the grain boundary structures and therefore remain unclear. A new physical model of plastic deformation is proposed in this letter to describe the cooperative process of nano-grain rotation and shear-coupled grain boundary migration as a new and effective toughening mechanism.

\section{Model description}

Consider the structural transformations associated with the cooperative process in a deformed elastically isotropic NC specimen. 
a

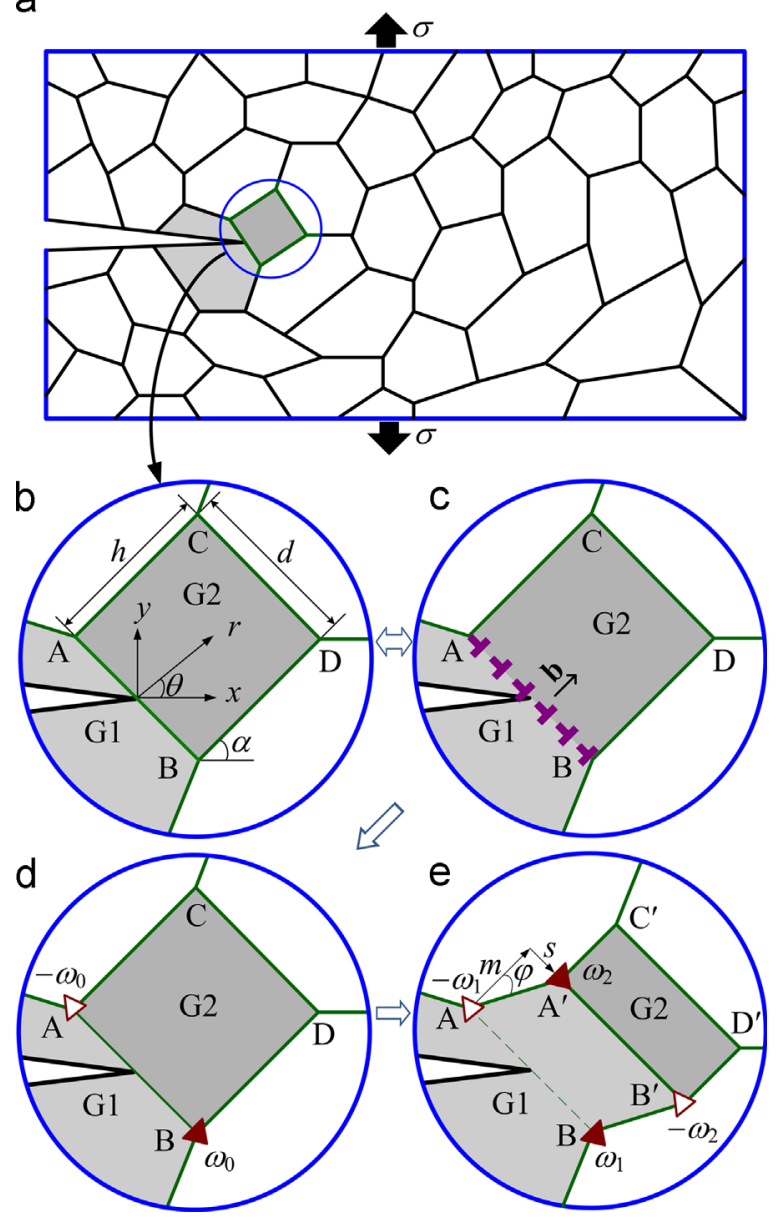

Fig. 1. Cooperative process of nano-grain rotation and shear-coupled migration of grain boundaries in a cracked nanocrystalline specimen: (a) schematic of a twodimensional microstructure; (b) initial configuration of the grain boundaries; (c) equivalent configuration of (b), where the grain boundary 'AB' is modeled as a finite array of discrete dislocations with identical Burgers vector b; (d) grains G1 and $\mathrm{G} 2$ rotate through GB dislocations climb, resulting in a disclination dipole with strength $\pm \omega_{0}$ (small triangles); and (e) following rotation, the motion of ' $\mathrm{AB}$ ' generates two disclination dipoles with strength $\pm \omega_{1}$ and $\pm \omega_{2}$.

A mode I crack is assumed to form in the $\mathrm{NC}$ material under a remote tensile stress $\sigma$ and for simplicity, the crack tip is assumed to locate at the center of a grain boundary 'AB' as shown in Fig. 1b. The grain boundary 'AB' is modeled as a finite wall of dislocations with identical Burgers vector b in Fig. 1c [18], which is characterized by a tilted misorientation parameter $\theta_{0}$ and the number of grain boundary dislocations $n_{0}$. Grains G1 and G2 rotate due to a sufficiently high stress induced by the crack, accompanying with the climb and dissociation of grain boundary dislocations, which results in a disclination dipole with an arm $d$ and strength $\pm \omega_{0}=(\mathrm{nb} / \mathrm{d})$ at the grain boundary 'AB' [12], as observed in experiments [19] (Fig. 1d). $n$ is the number of dissociated GB dislocations during NGR, which stands for the level of rotation. As a result of rotation, the misorientation parameter is reduced from $\theta_{0}$ to $\theta_{n}$, following a stimulated SCM process [20]. In the SCM process, the grain boundary 'AB' migrates with a normal distance $m$ and a tangential translation $s$ due to the shear deformation as shown in Fig. 1e. A coupling factor $\beta$ is introduced to define the ratio of $s$ to $m, \beta=s / m$ [5]. As a result, two disclination dipoles are generated during the combining process; one is $\mathrm{AB}$ with strength $\pm \omega_{1}= \pm \theta_{0}$ and the other is $\mathrm{A}^{\prime} \mathrm{B}^{\prime}$ with $\pm \omega_{2}= \pm\left(\theta_{0}-n b / d\right)$ as shown in Fig. 1e.

In order to consider the effect of two disclination dipoles on the fracture toughness of the nanocrystalline specimen, the criterion of a mode I brittle crack growth that is based on the balance between the energy release rate and the energy needed by a new free surface formation [21] is modified as follows:

$\frac{1-\nu}{2 G}\left(K_{I}^{2}+K_{I I}^{2}\right)=2 \gamma_{e}$,

where

$K_{I}=K_{\mathrm{I}}^{\sigma}+k_{\mathrm{I}}^{\mathrm{q}}, \quad K_{I I}=k_{\mathrm{II}}^{\mathrm{q}}$.

Here $K_{I}^{\sigma}$ is the stress intensity factor of mode I induced by the applied load $\sigma ; G$ is the shear modulus and $v$ the Poisson ratio; $\gamma_{e}$ is an effective surface energy. $\gamma_{e}=\gamma$ and $\gamma_{e}=\gamma-\gamma_{b} / 2$ for cases of crack propagating in a grain interior and along a grain boundary, respectively, where $\gamma$ is the specific surface energy and $\gamma_{b}$ the grain boundary energy; $k_{\mathrm{I}}^{\mathrm{q}}$ and $k_{\mathrm{II}}^{\mathrm{q}}$ are stress intensity factors generated by the locally disclination dipoles.

In the present model, the effect of a locally plastic flow on crack growth is accounted for by introducing an effective stress intensity factor $K_{I C}^{\mathrm{e}}$. Comparing the present model to that of brittle crack propagation, the critical condition for crack growth can be presented as: $K_{\mathrm{I}}^{\sigma}=K_{I C}^{\mathrm{e}}$. Substituting Eq. (2) into Eq. (1) and considering the present criterion yield

$K_{\mathrm{IC}}^{\mathrm{e}}=\sqrt{\left(K_{\mathrm{IC}}^{\sigma}\right)^{2}-\left(k_{\mathrm{IIC}}^{q}\right)^{2}}-k_{\mathrm{IC}}^{q}$,

where $K_{\mathrm{IC}}^{\sigma}=\sqrt{4 G \gamma_{e} /(1-\nu)}$ is the brittle fracture toughness of a disclination-free state. $k_{\mathrm{IIC}}^{q}=\left.k_{\mathrm{II}}^{q}\right|_{K_{1}^{\sigma}=K_{I C}^{\mathrm{e}}}$ and $k_{\mathrm{IC}}^{q}=\left.k_{\mathrm{I}}^{q}\right|_{K_{1}^{\sigma}=K_{I C}^{\mathrm{e}}}$. Following [22], the quantities $k_{\mathrm{I}}^{\mathrm{q}}$ and $k_{\mathrm{II}}^{\mathrm{d}}$ can be expressed as

$k_{\mathrm{I}}^{\mathrm{q}}=\frac{G \sqrt{d}}{2 \sqrt{2 \pi}(1-\nu)} \sum_{k=1}^{4}(-1)^{k-1} \omega_{k} \sqrt{r_{k}^{\prime}}\left(3 \cos \left(\theta_{k} / 2\right)+\cos \left(3 \theta_{k} / 2\right)\right)$
$k_{\mathrm{II}}^{\mathrm{q}}=\frac{G \sqrt{d}}{2 \sqrt{2 \pi}(1-\nu)} \sum_{k=1}^{4}(-1)^{k-1} \omega_{k} \sqrt{r_{k}^{\prime}}\left(\sin \left(\theta_{k} / 2\right)+\sin \left(3 \theta_{k} / 2\right)\right)$,

where $r_{k}^{\prime}=r_{k} / d . r_{k}, \theta_{k}$ are coordinates of the $k$ th disclination, where $k=1,2,3$, and 4 corresponds to disclination $\mathrm{B}, \mathrm{B}^{\prime}, \mathrm{A}^{\prime}$ and $\mathrm{A}$, respectively, and $\theta_{k} \in(-\pi, \pi)$; coordinates $\left(r_{k}^{\prime}, \theta_{k}\right)$ can be determined from Fig. $1 \mathrm{e}$ as $r_{1}^{\prime}=1 / 2, \quad \theta_{1}=\alpha-\pi / 2 ; \quad r_{2}^{\prime}$ $=\sqrt{1 / 4+\left(m^{\prime} / \cos \varphi\right)^{2}+\beta m^{\prime}}, \theta_{2}=\theta_{1}+\arccos \left[\left(0.5+\beta m^{\prime}\right) / r_{2}^{\prime}\right] ; r_{3}^{\prime}=$ $\sqrt{1 / 4+\left(m^{\prime} / \cos \varphi\right)^{2}-\beta m^{\prime}}, \quad \theta_{3}=\theta_{2}+\arccos \left[\left(r_{2}^{\prime 2}+r_{3}^{\prime 2}-1\right) / \quad\left(2 r_{2}^{\prime} r^{\prime}\right)\right]$ and $r_{4}^{\prime}=1 / 2, \theta_{4}=\alpha+\pi / 2, m^{\prime}=m / d, \varphi=\arctan \beta$.

\section{Results and discussion}

The cooperative effect of NGR and SCM on the critical stress intensity factor $K_{I C}^{\mathrm{e}}$ is predicted for a nanocrystalline Al sample, where the parameters are taken as $G=27 \mathrm{GPa}$ [23], $v=0.34$ [23], $\gamma=0.56 \mathrm{~J} \mathrm{~m}^{-2}$ [24], $\gamma_{b}=\gamma / 2$ [24] and $\mathrm{b} \approx 0.25 \mathrm{~nm}$. The other parameters are set as $m=1.5 \mathrm{~nm}, \alpha=-\pi / 2$. The case of a grain boundary crack is considered since cracks in a NC material were found to tend to nucleate and propagate along grain boundaries.

Fig. 2 presents the variation of the ratio $K_{I C}^{\mathrm{e}} / K_{\mathrm{IC}}^{\sigma}$ with respect to the normalized number of dissociated GB dislocations $n / n_{0}$ for various nanocrystalline Al samples, where the effects of coupling factor $\beta$ and misorientation parameter $\theta_{0}$ are investigated as shown in Fig. 2(a and b), respectively. An improved toughening behavior should be demonstrated if $K_{I C}^{\mathrm{e}} / K_{\mathrm{IC}}^{\sigma} \geq 1$. Fig. 2a gives the effective fracture toughness linearly increasing with the normalized number of dissociated GB dislocations. The maximum value of $K_{I C}^{\mathrm{e}} / K_{\mathrm{IC}}^{\sigma}$ can reach up to 4.12 under given parameters. In Fig. 2b, an increasing $\theta_{0}$ from 0.1 to 0.5 can improve $K_{I C}^{\mathrm{e}} / K_{\mathrm{IC}}^{\sigma}$ from 2.08 to 6.22 , an approximate enhancement of factor 3 . The above results indicate that the cooperative mechanism plays a very important role in toughening NC materials, which consist of grain boundaries of mostly high angles. i.e., $\theta_{0} \geq 0.26$. 

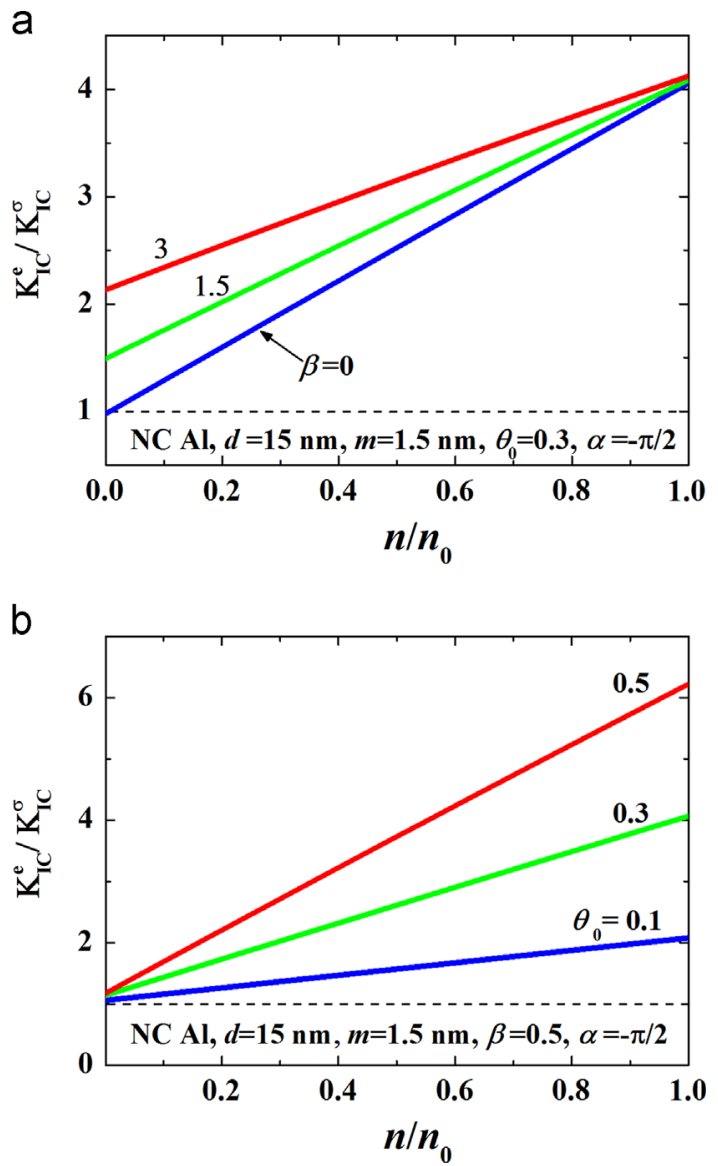

Fig. 2. Normalized critical stress intensity factor $K_{I C}^{\mathrm{e}} / K_{\mathrm{IC}}^{\sigma}$ as a function of the normalized number of dissociated GB dislocations $n / n_{0}$ for nanocrystalline Al considering the effects of coupling factor $\beta$ (a) and misorientation parameter $\theta_{0}$ (b).

\section{Conclusions}

In summary, a new physical model of plastic deformation is proposed based on the combination of two main nano-grain growth modes, i.e., nano-grain rotation and shear-coupled migration of grain boundaries. The cooperative behavior is demonstrated as a very effective toughening mechanism for nanocrystalline materials. Since both the coupling factor and misorientation parameter are governed by the specific structure of grain boundaries, it is possible to achieve an optimal balance between strength and toughness of a nanocrystalline material through grain boundary engineering.

\section{Acknowledgments}

J. Li acknowledges the financial support by the Start-up funding for the Newly-recruited High-level Talents from Northwestern Polytechnical University, China. S. Chen thanks the support from NSFC through Grants \#11125211 and 11372317 and the 973 Nanoproject (2012CB937500).

\section{References}

[1] Meyers MA, Mishra A, Benson DJ. Prog Mater Sci 2006;51:427-556.

[2] Gianola DS, Van Petegem S, Legros M, Brandstetter S, Van Swygenhoven H, Hemker KJ. Acta Mater 2006;54:2253-63.

[3] Fang TH, Li WL, Tao NR, Lu K. Science 2011;331:1587-90.

[4] Jin M, Minor AM, Stach EA, Morris JW. Acta Mater 2004;52:5381-7.

[5] Cahn JW, Mishin Y, Suzuki A. Acta Mater 2006;54:4953-75.

[6] Li J, Soh AK. Acta Mater 2013;61:5449-57.

[7] Trautt ZT, Adland A, Karma A, Mishin Y. Acta Mater 2012;60:6528-46.

[8] Molodov DA, Gorkaya T, Gottstein G. J Mater Sci 2011;46:4318-26.

[9] Gorkaya T, Burlet T, Molodov DA, Gottstein G. Scr Mater 2010;63:633-6.

[10] Gorkaya T, Molodov DA, Gottstein G. Acta Mater 2009;57:5396-405.

[11] Molodov DA, Gorkaya T, Gottstein G. Scr Mater 2011;65:990-3.

[12] Li J, Soh AK. Scr Mater 2014, http://dx.doi.org/10.1016/j.scriptamat.2013.12.021.

[13] Haslam AJ, Phillpot SR, Wolf H, Moldovan D, Gleiter H. Mater Sci Eng A 2001;318:293-312.

[14] Moldovan D, Wolf D, Phillpot SR, Haslam AJ. Acta Mater 2002;50:3397-414.

[15] Gorkaya T, Molodov KD, Molodov DA, Gottstein G. Acta Mater 2011;59:5674-80.

[16] Trautt ZT, Mishin Y. Acta Mater 2012;60:2407-24.

[17] Sansoz F, Dupont V. Appl Phys Lett 2006;89:111901.

[18] Sutton AP, Balluffi RW. Interfaces in crystalline materials. 1st ed.. New York: Oxford University Press; 1995.

[19] Liu P, Mao SC, Wang LH, Han XD, Zhang Z. Scr Mater 2011;64:343-6.

[20] Li J, Soh AK. Appl Phys Lett 2012;101:241915.

[21] Irwin G. J Appl Mech 1957;24:361-4.

[22] Li J, Soh AK. Scr Mater 2013;69:283-6.

[23] Gale WF, Totemeier TC. Smithells metals reference book. 8th ed.. Oxford: Butterworth-Heinemann; 2003.

[24] Zhu YT, Liao XZ, Srinivasan SG, Zhao YH, Baskes MI, Zhou F, et al. Appl Phys Lett 2004;85:5049-51. 